\title{
Adsorption and dissociation of hydrogen molecules on bare and functionalized carbon nanotubes
}

\author{
S. Dag, ${ }^{1}$ Y. Ozturk, ${ }^{1}$ S. Ciraci, ${ }^{1, *}$ and T. Yildirim ${ }^{2}$ \\ ${ }^{1}$ Department of Physics, Bilkent University, Ankara 06800, Turkey \\ ${ }^{2}$ NIST Center for Neutron Research, Gaithersburg, Maryland 20899, USA
}

(Received 12 March 2005; revised manuscript received 9 June 2005; published 6 October 2005)

\begin{abstract}
Interaction between hydrogen molecules and bare as well as functionalized single-wall carbon nanotubes (SWNT) is investigated using first-principles plane wave method. It is found that the binding energy of the $\mathrm{H}_{2}$ physisorbed on the outer surface of the bare SWNT is very weak, and cannot be enhanced significantly either by increasing the curvature of the surface through radial deformation, or by the coadsorption of a Li atom that makes the semiconducting tube metallic. Although the bonding is strengthened upon adsorption directly to the $\mathrm{Li}$ atom, its nature continues to be physisorption. However, the character of the bonding changes dramatically when SWNT is functionalized by the adsorption of a Pt atom. A single $\mathrm{H}_{2}$ is chemisorbed to the $\mathrm{Pt}$ atom on the SWNT either dissociatively or molecularly. The dissociative adsorption is favorable energetically and is followed by the weakening of the Pt-SWNT bond. Out of two adsorbed $\mathrm{H}_{2}$, the first one can be adsorbed dissociatively and the second one is chemisorbed molecularly. The nature of bonding is a very weak physisorption for the third adsorbed $\mathrm{H}_{2}$. Palladium also promotes the chemisorption of $\mathrm{H}_{2}$ with relatively smaller binding energy. Present results reveal the important effect of transition metal atom adsorbed on SWNT and these results advance our understanding of the molecular and dissociative adsorption of hydrogen for efficient hydrogen storage.
\end{abstract}

DOI: 10.1103/PhysRevB.72.155404

PACS number(s): 73.22.-f, 61.46.+w, 68.43.Bc

\section{INTRODUCTION}

Realization of fuel cells has been a real challenge for a clean and efficient source of energy in diverse fields of applications with different size and capacity range. Once hydrogen is chosen as a potential fuel, its storage, easy discharge for consumption and dissociation into hydrogen atoms in the fuel cell to produce the desired electromotive force involve several problems that need to be solved. Single-wall carbon nanotubes (SWNT) have been the focus of attention for hydrogen storage because of their high surface to volume ratios. Dillon et al. ${ }^{1}$ have pioneered the idea that nanotubes can be an efficient, cheap, and rechargeable storage medium for small-scale fuel cells and they estimated 5-10 weight percent (wp) $\mathrm{H}_{2}$ adsorption in SWNTs. Later, Ye et al. ${ }^{2}$ and Liu et al. ${ }^{3}$ obtained $\mathrm{H}_{2}$ storage capacities of 8.2 and $4.2 \mathrm{wp}$, respectively. Unfortunately, recent studies further exploring this idea have come up with controversial conclusions. ${ }^{4-9}$ In the meantime, adsorption of alkali atoms on SWNTs have been proposed to enhance the $\mathrm{H}_{2}$ uptake. ${ }^{9,10}$ Nevertheless, the functionalization of SWNTs to render them feasible for hydrogen storage through coverage of suitable adatoms still needs to be explored.

This paper ${ }^{11}$ clarifies controversial issues related to the storage of hydrogen molecules on carbon nanotubes. First we address the following questions: (i) $\mathrm{Can}$ a $\mathrm{H}_{2}$ molecule be adsorbed on SWNT? (ii) What is the nature and strength of the bonding? (iii) Can the strength of the bonding be modified either by changing the curvature of the surface or by the coadsorption of metal atoms? Then, we examined whether the functionalization of SWNTs by transition metal elements can promote the $\mathrm{H}_{2}$ uptake and give rise to the dissociation of $\mathrm{H}_{2}$. To answer all these questions we investigated the interaction between the $\mathrm{H}_{2}$ molecule and the bare, radially deformed, as well as foreign atom adsorbed SWNTs by carry- ing out calculations within the density functional theory (DFT). ${ }^{12}$ Our results not only advance our understanding of $\mathrm{H}_{2}$ adsorption on carbon nanotubes, but also suggest new ways for efficient hydrogen storage.

Our calculations have been carried out using a firstprinciples plane wave method and ultrasoft pseudopotentials ${ }^{13,14}$ within the generalized gradient approximation (GGA). ${ }^{15}$ Adsorption and dissociation of $\mathrm{H}_{2}$ is treated within the supercell geometry with lattice parameters $a_{S C}=20$ $\AA, b_{S C}=20 \AA$, and $c_{S C}=4.26 \AA$. The lattice parameter of the SWNT along its axis $c$ is taken to be equal to $c_{S C}$. In order to reduce the adsorbate-adsorbate interaction, certain systems are treated in double supercells with $c_{S C}=2 c$. All atomic positions (SWNT and adsorbates), as well as the lattice parameter of the supercell $c_{S C}$ are optimized by minimizing the total energy $E_{T}$, atomic forces, and the stress of the system. The Brillouin zone is sampled by $1 \times 1 \times 23$ special $\mathbf{k}$ points $(1 \times 1 \times 11$ for double cells) using the Monkhorst-Pack scheme. ${ }^{16}$ The calculations involving the graphite-adsorbate systems are carried out by $4 \times 4 \times 1$ special k-point sampling. Bloch wave functions are expanded by plane waves with the kinetic energy $\hbar^{2}|\mathbf{k}+\mathbf{G}|^{2} / 2 \mathrm{~m}<400 \mathrm{eV}$. The weak attractive Van der Waals ( $\mathrm{VdW}$ ) interaction becomes crucial in calculating binding energies of weak physisorption bonds, but is not well represented by DFT using GGA. ${ }^{17}$ Therefore, in the case of physisorption, the contribution of weak and attractive $\mathrm{VdW}$ interaction energy, $E_{V d W}$, to the binding energy is obtained from the Slater-Kirkwood approximation ${ }^{18}$ using the asymptotic form of Lifshitz's equation. ${ }^{19}$ This approach, however, is not suitable for determining the contribution of $\mathrm{VdW}$ interaction in the chemisorption of molecules. Nevertheless, in the chemisorption regime the VdW interaction is much weaker than the chemical interaction and hence is neglected in the discussion of chemisorption energy. 


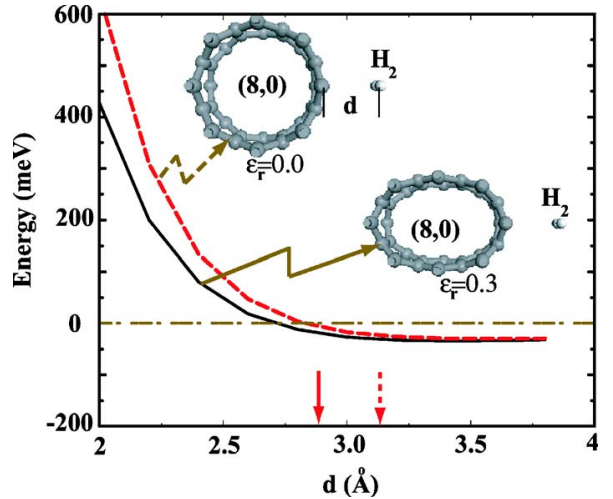

FIG. 1. (Color online) Variation of chemical interaction energy $E_{C}$ between SWNT and $\mathrm{H}_{2}$ molecule as a function of distance $d$ between them. Two cases, namely adsorption to bare and radially deformed SWNT, are shown by dashed and continuous lines, respectively. In calculating both curves, atomic structures corresponding to $d \rightarrow \infty$ have been used without relaxation. The dash-dotted line indicates zero of the chemical interaction energy. Optimized distance for the two cases are indicated by arrows.

In the present calculations we used the zigzag $(8,0)$ tube as a prototype for SWNT.

\section{ADSORPTION OF $\mathrm{H}_{2}$ ON BARE AND RADIALLY DEFORMED SWNT}

To clarify whether $\mathrm{H}_{2}$ can form stable bonding on the outer or inner surface of a SWNT, we calculated the chemical interaction energy between $\mathrm{H}_{2}$ and the outer surface of the $(8,0)$ SWNT (Ref. 20) at different sites (i.e., the $H$ site, above the hexagon; $Z$ and $A$ sites above the zigzag and above the axial $\mathrm{C}-\mathrm{C}$ bonds; the $T$ site, a bridge site between two adjacent zigzag $\mathrm{C}-\mathrm{C}$ bonds) as a function of spacing $d$. At all these sites the $\mathrm{H}_{2}$ molecule has remained parallel to the surface of SWNT. The chemical interaction energy is obtained from the expression, $E_{C}(d)=E_{T}\left[\mathrm{H}_{2}+\mathrm{SWNT}, d\right]$ $-E_{T}[\mathrm{SWNT}]-E_{T}\left[\mathrm{H}_{2}\right]$, in terms of the total energies of the bare nanotube $\left(E_{T}[\mathrm{SWNT}]\right)$, free $\mathrm{H}_{2}\left(E_{T}\left[\mathrm{H}_{2}\right]\right)$, and $\mathrm{H}_{2}$ attached to SWNT at a distance $d\left(E_{T}\left[\mathrm{H}_{2}+\mathrm{SWNT}, d\right]\right)$. Here $E_{C}<0$ corresponds to an attractive interaction. The stable binding occurs at the minimum of $E_{C}(d)+E_{V d W}(d)$; the negative of this sum is denoted as the binding energy $E_{b}$. The binding is exothermic when $E_{b}>0$. In Fig. 1 we show the variation of $E_{C}(d)$ calculated for unrelaxed atomic structures at the $H$ site. Once the atomic structures of both SWNT and $\mathrm{H}_{2}$ molecules are relaxed the minimum value of $E_{C}(d)$ is found to be $-27 \mathrm{meV}$ at $d_{0}=3.1 \AA$ at the $H$ site. Minimum values of $E_{C}(d)$ calculated for $A, Z$, and $T$ sites are also very small and comparable to that of the $H$ site. Han and Lee ${ }^{21}$ performed DFT calculations using the local basis set to calculate $E_{C}(d)$ between $\mathrm{H}_{2}$ and $(10,0)$ SWNT. They found that $E_{C}(d)$ of $\mathrm{H}_{2}$ with a vertical orientation at the $H$ site has a minimum value of $-34 \mathrm{meV}$ at $d_{0}=3.44 \AA$. However, they predicted the interaction energy $E_{C}(d)=-25 \mathrm{meV}$ for the configuration where the adsorbed $\mathrm{H}_{2}$ molecule is parallel to the surface at the $H$ site. We calculated the long range
VdW interaction energy for the $H$ site at $d_{0}=3.1 \AA$ to be $E_{V d W} \sim-30 \mathrm{meV}$. Then the binding energy associated with the $\mathrm{H}_{2}$ molecule adsorbed at the $H$ site is calculated to be $E_{b} \sim 57 \mathrm{meV}$ in the present study. This is a small binding energy and indicates physisorption. ${ }^{22}$

Previous studies ${ }^{4,5}$ have revealed that the physisorption of individual $\mathrm{H}_{2}$ molecules with $E_{b}>0$ cannot occur on the inner wall of SWNT. On the contrary, Han and Lee ${ }^{21}$ obtained the lowest chemical interaction energy $E_{C}\left(d_{0}\right)$ $=-60 \mathrm{meV}$ for a vertical $\mathrm{H}_{2}$ bound at the inner $H$ site of $(10,0)$ SWNT. Hydrogen molecules preferred to stay either at the center of the tube or form some cylindrical shells inside depending on radius of the tube. For the $(8,0)$ tube we found that $\mathrm{H}_{2}$ is trapped and stabilized at the center of the tube with a repulsive interaction energy $E_{C}=+9 \mathrm{meV}$. Repulsive interaction arises since $R<d_{0}$. We note that the implementation of $\mathrm{H}_{2}$ inside the tube having radius in the range of $3 \AA$ is expected to be hindered by this repulsive interaction.

Earlier it has been shown that the binding energy of foreign atoms adsorbed on SWNT increases with increasing curvature. $^{23}$ Tada et al. $^{24}$ have argued that the potential barrier associated with the dissociative adsorption of $\mathrm{H}_{2}$ on SWNT is lowered with increasing curvature of the tube. It has been proposed that the potential barrier for the dissociation of $\mathrm{H}_{2}$ adsorbed in the interstitial region between tubes can be lowered by applying radial deformation to the rope or to $\mathrm{SWNT}^{25}$ It is known that under radial deformation the circular cross section changes and consequently the curvature varies at different locations on the surface. Motivated with these effects of curvature, we examine whether the attractive interaction energy $E_{C}$ can be enhanced by changing the curvature of the tube via radial deformation. Radial deformation is realized by pressing the tube between two ends of a given diameter. This, in turn, changes the circular cross section of the bare tube with radius $R_{0}$ to an elliptical one with major and minor axes $2 a$ and $2 b$, respectively. The atomic structure of the $(8,0)$ tube is optimized under compressive radial strain $\epsilon_{r}=\left(b-R_{0}\right) / R_{0} \simeq-0.3$ by fixing the rows of carbon atoms at the both ends of minor axis. The deformation is reversible so that the tube goes back to its original circular form upon the release of radial strain. ${ }^{26}$ The deformation energy (that is the difference between the total energies of deformed and undeformed SWNTs) is calculated to be $E_{D}=1.4 \mathrm{eV}$ per unit cell. We examined whether the binding energy of the $\mathrm{H}_{2}$ molecule changes under the radial deformation of SWNT. Figure 1 shows the variation of $E_{C}(d)$ for $\mathrm{H}_{2}$ approaching toward the high curvature site of the tube (i.e., one end of the major axis) at the $H$ site. The minimum value of the attractive interaction, $E_{C}$ is $-30 \mathrm{meV}$ and occurs at $d_{0}=2.9 \AA$. This is only $3 \mathrm{meV}$ stronger than that of $\mathrm{H}_{2}$ physisorbed to the undeformed (circular) tube. Hence, the enhancement of the binding energy of $\mathrm{H}_{2}$ due to curvature effect is negligibly small due to a relatively large value of $d_{0}$. Our result suggests that the physisorption energy does not vary significantly with the radius of SWNT. The minimum value of $E_{C}(d)$ is small and does not vary significantly relative to the adsorption site ( $H, A, Z$, and $T$ sites). This also implies that the chirality of the tube has negligible effects on the physisorption energy. Apparently, the binding of $\mathrm{H}_{2}$ on the outer surface of SWNT is weak and the corresponding 


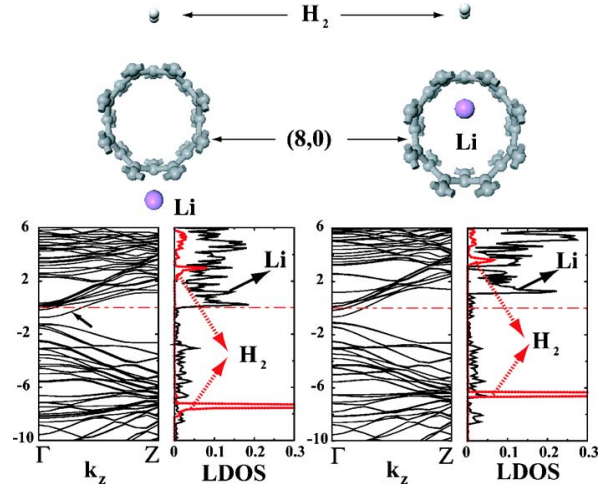

FIG. 2. (Color online) Atomic configuration, energy band structure, and local density of states (LDOS) calculated for the coadsorption of the $\mathrm{H}_{2}$ molecule and a single Li atom. Two cases correspond to $\mathrm{Li}$ atom chemisorbed on the external and internal surface of the $(8,0)$ zigzag SWNT. Zero energy is set at the Fermi level $E_{F}$. LDOS calculated at $\mathrm{Li}$ and $\mathrm{H}_{2}$ are shown by continuous and dotted lines, respectively. Metallized SWNT bands are indicated by arrows.

physisorption energy is small. The binding cannot be enhanced significantly by increasing the curvature locally through radial deformation. Curvature effects or radial deformation may be significant at small $d$ when $\mathrm{H}_{2}$ is forced towards the SWNT surface. Present results are in line with the work by Kostov et al. ${ }^{27}$

\section{COADSORPTION OF HYDROGEN MOLECULE AND LITHIUM ATOM ON SWNT}

The binding of $\mathrm{H}_{2}$ may be enhanced by the coadsorbed foreign atoms. We first consider a $\mathrm{Li}$ atom adsorbed on the $(8,0)$ SWNT, since the adsorption of an alkali atom has been proposed to enhance the $\mathrm{H}_{2}$ uptake. ${ }^{9,10}$ The Li atom is chemisorbed at the $H$ site, $1.5 \AA$ above the surface of SWNT with a binding energy of $0.8 \mathrm{eV}$. Self-consistently calculated electronic band structure shown in Fig. 2 reveals that chemisorbed $\mathrm{Li}$ atoms donate their $2 s$ valence electrons to the lowest conduction $\pi^{*}$ band so that the semiconducting $(8,0)$ SWNT (having a band gap of $E_{g}=0.6 \mathrm{eV}$ ) becomes metallic. This is a behavior common to the other alkali atoms adsorbed on SWNTs. ${ }^{28}$ In order to examine the indirect effect of coadsorbed $\mathrm{Li}$ we consider $\mathrm{H}_{2}$ as attached to the opposite site of the adsorbed $\mathrm{Li}$ atom. The optimized structure of the physisorbed $\mathrm{H}_{2}$ is shown in Fig. 2 together with the relevant structural parameters. We found that $E_{C}$ has a minimum value of $-35 \mathrm{meV}$ at $d_{0}=3.4 \AA$. A similar calculation has been also performed for a $\mathrm{Li}$ atom adsorbed on the inner wall of SWNT while $\mathrm{H}_{2}$ is on the external wall directly above the coadsorbed Li as shown in Fig. 2. In this adsorption configuration the minimum value of $E_{C}$ practically did not change. The local density of states calculated on the Li atom and $\mathrm{H}_{2}$ refuses the possibility of any significant interaction between adsorbates. As a result, our calculations for both external and internal adsorption of Li rule out significant indirect effect of coadsorbed $\mathrm{Li}$ to enhance the binding of $\mathrm{H}_{2}$ on SWNT. The occupation of empty conduction bands by the alkali electrons and hence metallization of SWNT did not affect the bonding

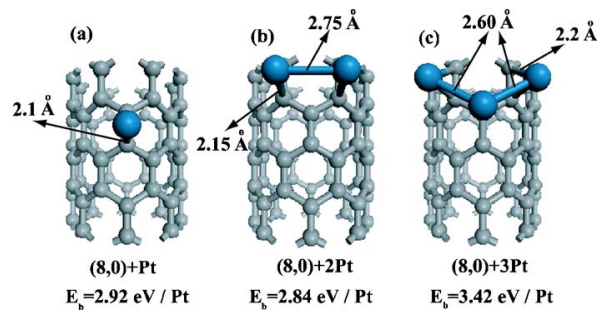

FIG. 3. (Color online) (a) Atomic configurations for single, double, and triple Pt atoms adsorbed on the $(8,0)$ SWNT. Average binding energies of adsorbed Pt atoms $E_{b}$ and bond distances are indicated.

of $\mathrm{H}_{2}$. These results are in agreement with the first principles calculations by Lee et al. ${ }^{29}$ However, the effect of Li on the adsorption of $\mathrm{H}_{2}$, whereby $\mathrm{H}_{2}$ is attached directly to $\mathrm{Li}$ atom is found significant. The minimum value of $E_{C}$ has dropped to $-175 \mathrm{meV}$ (i.e., $E_{b}$ becomes stronger), while $d_{0}$ decreases to $2.1 \AA$. Clearly, the energy associated with the direct binding of $\mathrm{H}_{2}$ to $\mathrm{Li}$ is enhanced, but the nature of bonding remains as physisorption.

\section{COADSORPTION OF HYDROGEN MOLECULE AND PLATINUM ATOM ON SWNT}

A single transition metal atom adsorbed on the outer surface of SWNT has shown interesting properties, such as high bind energy and magnetic ground states with high net magnetic moment. For example, transition element atoms (Ti, V, $\mathrm{Cr}, \mathrm{Mn}, \mathrm{Fe}, \mathrm{Co}, \mathrm{Pt}$, etc.) have crucial adsorption states on nanotubes ${ }^{28}$ and some of them (Ti, Ni, Pd) form continuous or quasicontinuous metal coatings on the SWNT. ${ }^{30,31}$ As for the Pt atom, it is known to be a good catalyst in various chemical processes. While SWNTs offer high surface to volume ratio, the interaction between $\mathrm{H}_{2}$ and $\mathrm{Pt}$ atom adsorbed on SWNT may be of interest. Now we investigate the character of the bonding between $\mathrm{H}_{2}$ and Pt adsorbed on SWNT and address the question of how many $\mathrm{H}_{2}$ molecules can be attached to an adsorbed Pt atom and how strong is the binding.

\section{A. Adsorption of Pt atoms on SWNT}

We first examine the adsorption of Pt atom(s) on $(8,0)$ SWNT. The character of the bonding has been investigated first by placing the $\mathrm{Pt}$ atoms on the $A$ sites of the $(8,0)$ tube (that is known to yield highest binding energy ${ }^{28}$ ) and subsequently by optimizing the whole structure. Three different adsorption configurations have been examined, namely one, two, and three Pt atoms adsorbed at the adjacent sites. The latter represents a small cluster on SWNT as described in Fig. 3. Calculated binding energies of the Pt atoms increase as the number of $\mathrm{Pt}$ atoms increases from one to three in the same neighborhood. On the other hand, the $\mathrm{C}$-Pt distance also gradually increases with an increasing number of Pt atoms adsorbed in the same neighborhood. This paradoxical situation can be understood by the increasing Pt-Pt coupling, that happens to retract electronic charge from the $\mathrm{C}$ - Pt bonds derived by the $\mathrm{Pt} 3 d$ and $\mathrm{C} 2 p$ orbitals. 

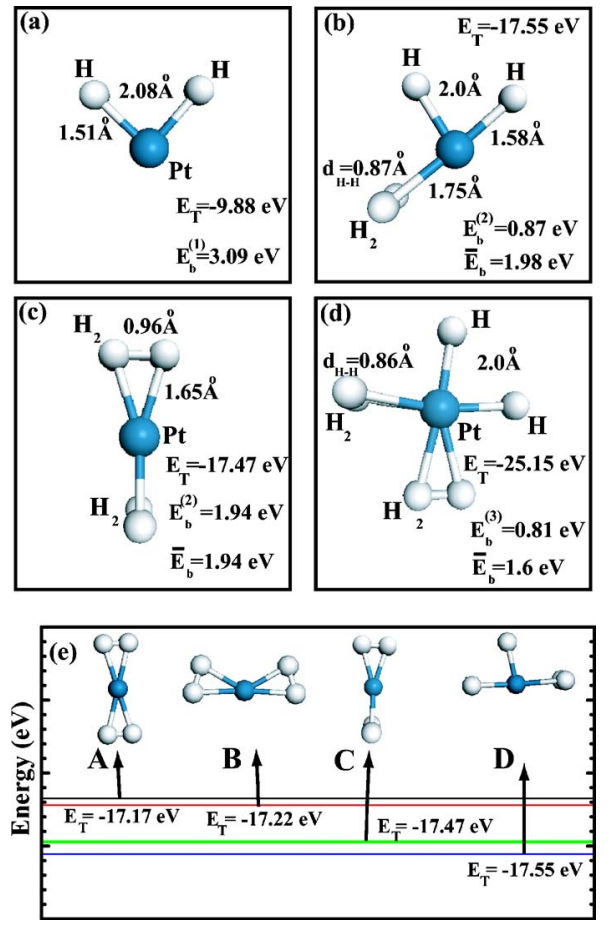

FIG. 4. (Color online) Optimized binding configuration of $\mathrm{H}_{2}$ molecules adsorbed to a free Pt atom. (a) Dissociative adsorption of a single $\mathrm{H}_{2}$ molecule. (b) The first $\mathrm{H}_{2}$ is dissociatively, second $\mathrm{H}_{2}$ molecularly adsorbed. (c) Two $\mathrm{H}_{2}$ are molecularly adsorbed. (d) Two $\mathrm{H}_{2}$ are molecularly, one $\mathrm{H}_{2}$ dissociatively adsorbed. (e) Four different configurations related with the adsorption of two $\mathrm{H}_{2}$ to the same free $\mathrm{Pt}$ atom. Binding energy of the $n$th $\mathrm{H}_{2}$ molecule adsorbed to $\mathrm{Pt}$ atom, $E_{b}^{(n)}$; average binding energy per $\mathrm{H}_{2}, \bar{E}_{b}$; total energy with respect to constituent atoms, $E_{T}$; various bond distances are indicated.

\section{B. Adsorption of $\mathrm{H}_{2}$ to a free $\mathrm{Pt}$ atom}

Optimized structures of $\mathrm{H}_{2}$ adsorbed to a free $\mathrm{Pt}$ atom are shown in Fig. 4. Upon approaching a free Pt atom, a single $\mathrm{H}_{2}$ molecule dissociates and forms $\mathrm{PtH}_{2}$ with the $\mathrm{Pt}-\mathrm{H}$ bond distance $d_{\mathrm{Pt}-\mathrm{H}}=1.51 \AA$ and $d_{\mathrm{H}-\mathrm{H}}=2.08 \AA$. The total energy $E_{T}$ relative to the energies of free $\mathrm{Pt}$ and $\mathrm{H}$ atoms is calculated as $-9.88 \mathrm{eV}$. The binding energy relative to the $\mathrm{H}_{2}$ molecule and the free $\mathrm{Pt}$ atom (namely, $E_{b}^{(1)}=E_{T}\left[\mathrm{H}_{2}\right]+E_{T}[\mathrm{Pt}]$ $\left.-E_{T}\left[\mathrm{PtH}_{2}\right]\right)$ is $3.09 \mathrm{eV}$. The weak $\mathrm{VdW}$ interaction is not included in $E_{b}$, since $\left|E_{C}\left(d_{0}\right)\right| \gg\left|E_{V d W}\left(d_{0}\right)\right|$. As for the adsorption of additional $\mathrm{H}_{2}$ to $\mathrm{PtH}_{2}$, there are several minima on the Born-Oppenheimer surface determined by the conjugategradient method: The first minimum corresponds to a configuration given in Fig. 4(b) where $\mathrm{PtH}_{2}$ preserves the dissociated configuration while the second $\mathrm{H}_{2}$ is molecularly adsorbed. Even if the $\mathrm{H}-\mathrm{H}$ interaction of adsorbed $\mathrm{H}_{2}$ is weakened and hence the $\mathrm{H}-\mathrm{H}$ distance has increased to $0.87 \AA$, we still consider it as a molecular adsorption. We identify this configuration as $\mathrm{PtH}_{2}-\mathrm{H}_{2}$. The adsorbed $\mathrm{H}_{2}$ molecule is perpendicular to the plane of $\mathrm{PtH}_{2}$. The binding energy of the second $\mathrm{H}_{2}$ adsorbed to $\mathrm{PtH}_{2}$ (namely, $\left.E_{b}^{(2)}=E_{T}\left[\mathrm{H}_{2}\right]+E_{T}\left[\mathrm{PtH}_{2}\right]-E_{T}\left[\mathrm{PtH}_{2}-\mathrm{H}_{2}\right]\right)$ is calculated to be $0.87 \mathrm{eV}$. Under these circumstances the average binding energy per $\mathrm{H}_{2}$ is $\bar{E}_{b}=1.98 \mathrm{eV}$ for $\mathrm{PtH}_{2}-\mathrm{H}_{2}$. In the second con- figuration, identified as Pt- $2 \mathrm{H}_{2}$ as shown in Fig. 4(c), both $\mathrm{H}_{2}$ are molecularly adsorbed. As the $\mathrm{H}-\mathrm{H}$ molecular bonds are weakened, $d_{\mathrm{H}-\mathrm{H}}$ is increased to $0.96 \AA$ and all Pt-H bonds have a uniform length with $d_{\mathrm{Pt}-\mathrm{H}}=1.65 \AA$. Here adsorbed $\mathrm{H}_{2}$ molecules are perpendicular. The binding energy of each molecule is calculated to be $1.94 \mathrm{eV}$ slightly less than the average binding energy in the $\mathrm{PtH}_{2}-\mathrm{H}_{2}$ configuration. The configuration $\mathrm{PtH}_{2}-2 \mathrm{H}_{2}$ shown in Fig. 4(d) involves the adsorption of three $\mathrm{H}_{2}$ molecules; one is dissociatively, the remaining two are molecularly adsorbed. Here $\mathrm{Pt}-\mathrm{H}_{2}$ planes of two molecularly adsorbed $\mathrm{H}_{2}$ are perpendicular. The binding energy of the third $\mathrm{H}_{2}$ relative to the energy of $\mathrm{PtH}_{2}-\mathrm{H}_{2}$ in Fig. 4(b) is found to be $E_{b}^{(3)}=0.81 \mathrm{eV}$. Accordingly, the average binding energy of each $\mathrm{H}_{2}$ is $\bar{E}_{b}=1.6 \mathrm{eV}$ relative to the free $\mathrm{H}_{2}$ and free $\mathrm{Pt}$ atom. Figure 4(e) compares four distinct configurations related with the adsorption of two $\mathrm{H}_{2}$ molecules on the same free Pt atom. It appears that these configurations determined by the conjugate gradient method correspond to local energy minima and the configuration in Fig. 4(b) appears to have the lowest energy.

\section{Adsorption of $\mathrm{H}_{2}$ to a $\mathrm{Pt}$ atom on SWNT}

We deduced two configurations in the binding of a single $\mathrm{H}_{2}$ molecule to a $\mathrm{Pt}$ atom adsorbed on the $(8,0)$ SWNT as described in Fig. 5. While these two chemisorption configurations look dramatically different, their total energies differ by only $20 \mathrm{meV}$. In the configuration described in Fig. $5(\mathrm{a}), \mathrm{H}_{2}$ is dissociatively adsorbed with a binding energy $\left(E_{b}^{(1)}=E_{T}\left[\mathrm{H}_{2}\right]+E_{T}[\mathrm{SWNT}+\mathrm{Pt}]-E_{T}[\mathrm{SWNT}+\mathrm{Pt}+2 \mathrm{H}]\right) \quad$ of $1.18 \mathrm{eV}$. The $\mathrm{H}-\mathrm{H}$ and $\mathrm{Pt}-\mathrm{C}$ distances are 1.86 and $2.3 \AA$, respectively. In the configuration shown in Fig. 5(b), $\mathrm{H}_{2}$ is "molecularly" adsorbed in spite of the fact that the $\mathrm{H}-\mathrm{H}$ bond is significantly weakened. $\mathrm{H}_{2}$ approaching from different directions and angles results in a chemisorption state with binding energy $E_{b}=1.16 \mathrm{eV}$ and a Pt-H distance of $1.7 \AA$. The length of the $\mathrm{H}-\mathrm{H}$ bond has increased from 0.75 to $0.95 \AA$ upon adsorption. ${ }^{32}$ As compared to the configuration of dissociative adsorption in Fig. 5(a), the Pt-SWNT bond in the molecular adsorption of $\mathrm{H}_{2}$ in Fig. 5(b) is relatively stronger with a shorter bond length, $d_{\mathrm{Pt}-\mathrm{C}}=2.1 \AA$. Notably, while in the first configuration leading to dissociative adsorption in Fig. 5(a), the Pt atom is located near the hollow $H$ site; in the molecular adsorption of $\mathrm{H}_{2}$ in Fig. 5(b) the Pt atom is adsorbed at the $A$ site. We also note that the PtSWNT bond in the configurations described in Fig. 5(a) causes the binding energy to be smaller than the binding energy in Fig. 4(a).

Adsorption of $\mathrm{H}_{2}$ to a single $\mathrm{Pt}$ atom attached to the surface of graphite is of interest because it reveals how the binding energy and binding configuration of $\mathrm{H}_{2}$ depends on the radius of SWNT. We consider two configurations, namely, a single Pt atom is adsorbed near the hollow $H$ site, as shown in Fig. 5(c), and a Pt atom at the $A$ site as shown in Fig. 5(d). For both locations of the Pt atom on the graphite surface, a $\mathrm{H}_{2}$ molecule approaching the adsorbed $\mathrm{Pt}$ atom is dissociated and eventually forms two $\mathrm{Pt}-\mathrm{H}$ bonds. In this case the binding of $\mathrm{Pt}$ to the graphite surface is weaker than 

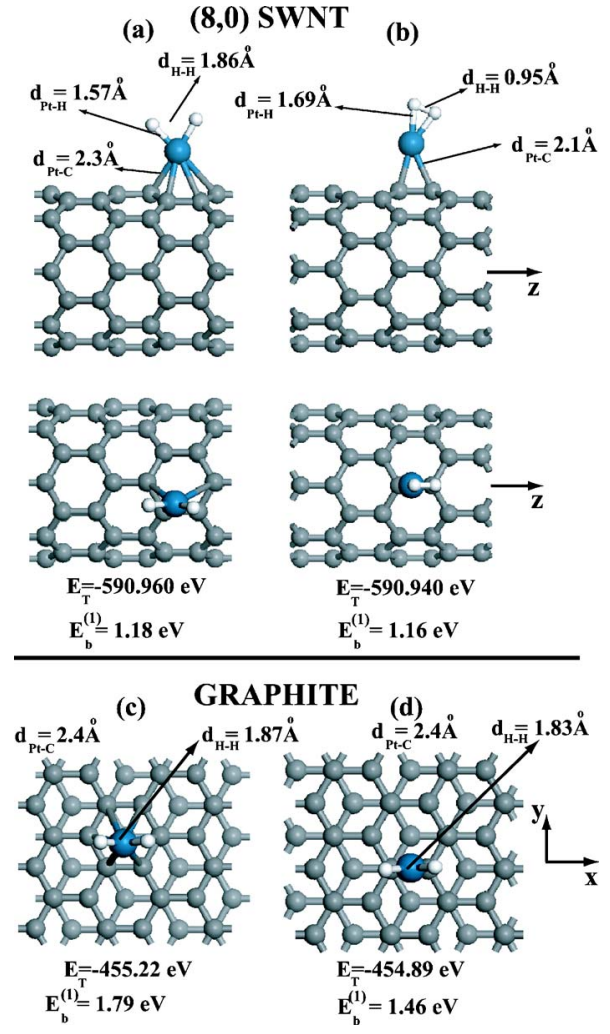

FIG. 5. (Color online) Optimized geometry for a single $\mathrm{H}_{2}$ molecule adsorbed to a single Pt atom. (a) The Pt atom is adsorbed near the $H$ site of $(8,0)$ SWNT (side and top view), (b) Pt at the $A$ site (bridge position) of $(8,0)$ SWNT (side and top view), (c) the Pt atom is adsorbed near the $H$ site of the graphite surface, and (d) the Pt atom at the $A$ site of graphite. $E_{T}$ is the total energy relative to the constituent free $\mathrm{C}, \mathrm{Pt}$, and $\mathrm{H}$ atoms.

that on the SWNT, and thus $d_{\mathrm{Pt}-\mathrm{C}}$ is increased to $2.4 \AA$. Relatively weaker interaction between $\mathrm{Pt}$ and graphite surface allows stronger interaction between $\mathrm{H}_{2}$ and $\mathrm{Pt}$, as in the case of a free Pt atom, and hence leads to the dissociation of the molecule. In view of the two limiting cases (i.e., small-radius SWNT versus graphene) in Fig. 5, one can expect that the dissociation of $\mathrm{H}_{2}$ may be favored if $\mathrm{Pt}$ is adsorbed on SWNTs having large $R$.

A systematic study outlined in Fig. 6 deals with the question of how many $\mathrm{H}_{2}$ molecules can be attached to a single $\mathrm{Pt}$ atom adsorbed on a SWNT. First we let a second $\mathrm{H}_{2}$ approach the Pt atom that has already one $\mathrm{H}_{2}$ molecule attached to it as in Fig. 5(b). The final optimized geometry of Pt and adsorbed $\mathrm{H}_{2}$ molecules in Fig. 6(a) is similar to the configuration of $\mathrm{PtH}_{2}-\mathrm{H}_{2}$ described in Fig. 4(b). First $\mathrm{H}_{2}$, which was initially chemisorbed to $\mathrm{Pt}$ as a molecule (with relatively increased $d_{\mathrm{H}-\mathrm{H}}$ ), has dissociated upon the molecular adsorption of the second $\mathrm{H}_{2}$. The dissociation of $\mathrm{H}_{2}$ is an indirect process and is mediated by the weakening of the Pt- $\mathrm{C}$ bonding upon the molecular adsorption of the second $\mathrm{H}_{2}$. The charge density difference $\Delta \rho(\mathbf{r})=\rho_{\mathrm{T}}(\mathbf{r})-\rho_{\mathrm{SWNT}}(\mathbf{r})$ $-\rho_{\mathrm{PtH}_{2}+\mathrm{H}_{2}}(\mathbf{r})$ calculated from the difference of total charge density $\rho_{T}(\mathbf{r})$ of SWNT $+\mathrm{PtH}_{2}+\mathrm{H}_{2}$ in Fig. 6(a) and those of the SWNT and the $\mathrm{PtH}_{2}+\mathrm{H}_{2}$ indicates that while the charge of Pt $d_{x y}$ and $\mathrm{C} p_{x, y}$ orbitals are depopulated, the Pt $d_{z^{2}}$ and $\mathrm{C}$
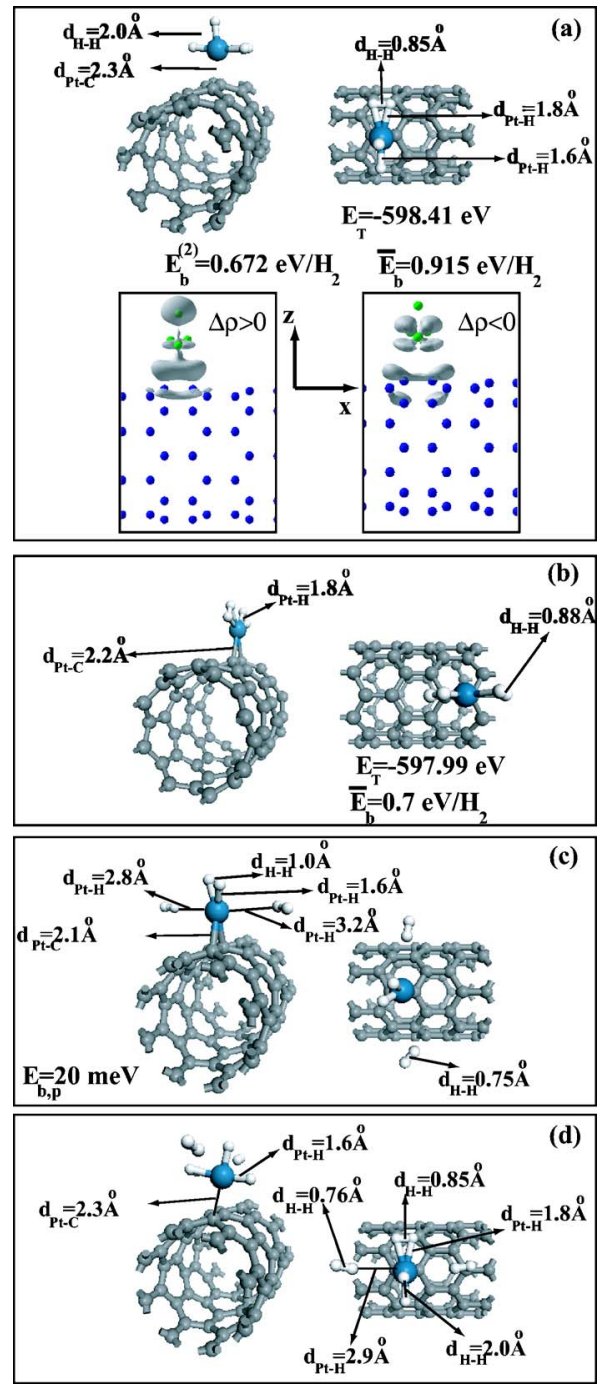

FIG. 6. (Color online) Optimized structure of $\mathrm{H}_{2}$ molecules adsorbed to the Pt atom on the SWNT. (a) One $\mathrm{H}_{2}$ adsorbed to $\mathrm{PtH}_{2}$ with a binding energy $E_{b}^{(2)}$. The inset shows the regions of charge depletion $(\Delta \rho<0)$ and charge accumulation $(\Delta \rho>0)$ as a result of the bonding between SWNT and $\mathrm{PtH}_{2}+\mathrm{H}_{2}$ in (a). (b) Another local minima where two $\mathrm{H}_{2}$ are molecularly adsorbed to the $\mathrm{Pt}$ atom with the average binding energy per $\mathrm{H}_{2}, \bar{E}_{b}$. (c) One $\mathrm{H}_{2}$ is chemisorbed, two $\mathrm{H}_{2}$ are weakly bound. (d) One $\mathrm{H}_{2}$ is dissociatively adsorbed, one $\mathrm{H}_{2}$ is molecularly chemisorbed, one $\mathrm{H}_{2}$ is physisorbed, and the fourth $\mathrm{H}_{2}$ escaped. The physisorption energy of $\mathrm{H}_{2}$ with $d_{\mathrm{Pt}-\mathrm{H}_{2}}$ $=3.2 \AA$ is $E_{b, p}$.

$p_{z}$ orbitals become populated to form the Pt-SWNT bond. This result suggests that in a reverse situation the weakening of the Pt-SWNT bond would lead to the transfer of charge from $\mathrm{Pt}-\mathrm{C}$ to $\mathrm{Pt}-\mathrm{H}$ bonds resulting in increased population of $d_{x y}$ orbitals that favors the dissociation of $\mathrm{H}_{2}$. Interestingly, exactly the same configuration has been obtained even when two $\mathrm{H}_{2}$ molecules approach concomitantly the bare Pt adsorbed on the SWNT. We note that the atomic configuration of a $\mathrm{PtH}_{2}-\mathrm{H}_{2}$ complex in Fig. 6(a) does not change significantly if SWNT is removed and the remaining system is relaxed. Another configuration related to the molecular adsorbtion of two $\mathrm{H}_{2}$ molecules is shown in Fig. 6(b). This 


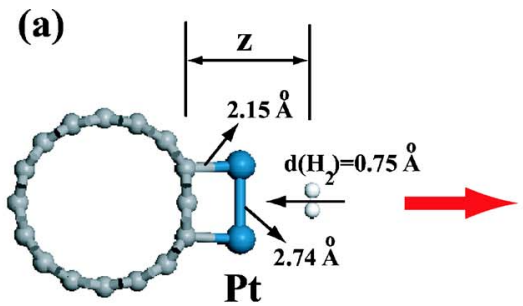

$(\mathbf{8 , 0})+2 \mathrm{Pt}$ (b)

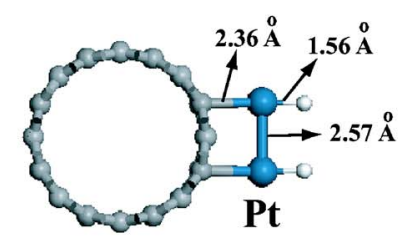

$\mathbf{P t}$ (d)

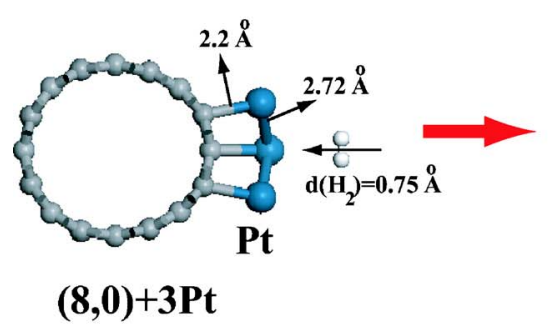

(e)

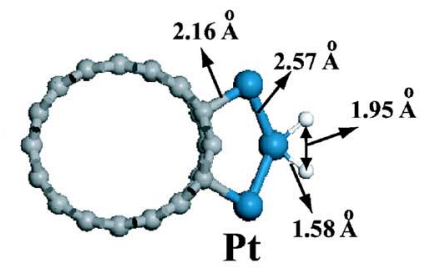

(c)

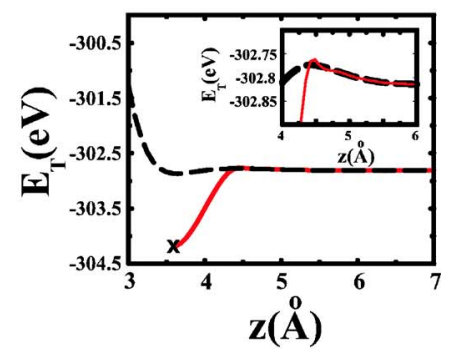

(f)

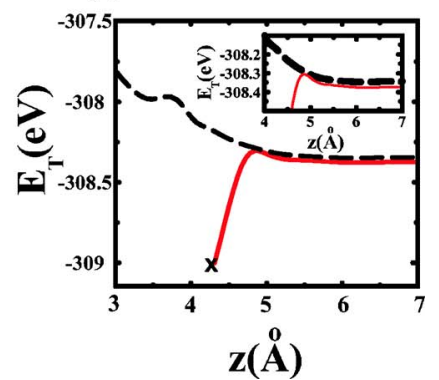

FIG. 7. (Color online) Dissociative adsorption of a single $\mathrm{H}_{2}$ molecule on a small Pt cluster adsorbed on $\mathrm{SWNT}$ (a). One $\mathrm{H}_{2}$ is approaching two adjacent Pt atoms adsorbed on SWNT. (b) Optimized geometry after dissociative adsorption of $\mathrm{H}_{2}$. (c) Variation of the total energy with distance $z$. Dashed curve corresponds to $E_{T}(z)$ for unrelaxed $\mathrm{H}_{2}$ and unrelaxed SWNT. Continuous curve corresponds to $E_{T}(z)$ of the relaxed geometry. (d), (e), and (f) are the same as (a), (b), and (c) except that the Pt cluster consists of three Pt atoms. $z$ is the distance from the surface of SWNT. Variation of $E_{T}(z)$ is amplified by inset.

appears to be a local minimum on the Born-Oppenheimer surface and has a binding energy $\sim 0.4 \mathrm{eV}$ less than that in Fig. 6(a).

In Fig. 6(c), two $\mathrm{H}_{2}$ approaching from both sides of the $\mathrm{PtH}_{2}$ on SWNT in Fig. 5(b) have been attached by weak physisorption bonds resulting in a $\mathrm{PtH}_{2}+2 \mathrm{H}_{2}$ configuration. Their distances to the $\mathrm{Pt}$ atom are relatively larger $\left(d_{\mathrm{Pt}_{-} \mathrm{H}_{2}}=2.1\right.$ and $\left.3.2 \AA\right)$ than the distance that occurred for molecularly chemisorbed $\mathrm{H}_{2}$. In particular, the latter $\mathrm{Pt}-\mathrm{H}_{2}$ distance is too long and the corresponding binding energy is only $\sim 20 \mathrm{meV}$. Even if its binding energy can increase slightly by the VdW interaction, the adsorbed $\mathrm{H}_{2}$ molecule can desorb and escape from Pt at room temperature. Note that due to the weak interaction between Pt and both physisorbed $\mathrm{H}_{2}$ molecules in Fig. 6(c) the $\mathrm{H}-\mathrm{H}$ distance of $\mathrm{PtH}_{2}$ remained small and consequently the $\mathrm{Pt}-\mathrm{C}$ bond retained its strength with a relatively smaller Pt-C distance. Attempts to attach more than three molecules to the Pt atom have failed. For example, as shown in Fig. 6(d), out of the four $\mathrm{H}_{2}$ molecules brought at the close proximity of the $\mathrm{Pt}$ atom, only three were attached (one being dissociatively chemisorbed, the second molecularly chemisorbed, and the third one physisorbed but the forth escaped). At the same time the PtSWNT bond has weakened and hence the $d_{\mathrm{Pt}-\mathrm{C}}$ distance has increased to $2.3 \AA$. Furthermore, we calculated the interaction energy between different $\mathrm{Pt}+\mathrm{H}_{2}$ complexes [namely $\mathrm{PtH}_{2}-\mathrm{H}_{2}$ in Fig. 6(a); Pt-2 $\mathrm{H}_{2}$ in Fig. 6(b); $\mathrm{PtH}_{2}-2 \mathrm{H}_{2}$ in Figs. 6(c)] and SWNT. Here the total energies are calculated using the same atomic structures as those in Figs. 6(a)-6(c). Calculated interaction energies for each case are $0.68,1.88$, and $0.78 \mathrm{eV}$, respectively. By using the similar procedure we also calculated the interaction energy between $\mathrm{Pt}+\mathrm{H}_{2}$ (where $\mathrm{H}_{2}$ is molecularly adsorbed) and SWNT in Fig. 5(b) to be $1.93 \mathrm{eV}$. Clearly, the variation of these energies with adsorbate structure and $\mathrm{Pt}-\mathrm{C}$ distances confirm the above arguments that the dissociation of one of the $\mathrm{H}_{2}$ molecules is followed by the weakening of the bond between $\mathrm{Pt}$ and SWNT.

\section{Adsorption of $\mathrm{H}_{2}$ to a small Pt cluster on SWNT}

As shown in Figs. 7(a) and 7(b), the situation is different in the case of the interaction between $\mathrm{H}_{2}$ and a small $\mathrm{Pt}$ cluster (consisting of a few Pt atoms adsorbed at close proximity). As $\mathrm{H}_{2}$ approaches two Pt atoms on SWNT, $\mathrm{H}_{2}$ starts to dissociate at a distance $\sim 3.9 \AA$ from the surface of SWNT. The optimized configuration is shown in Fig. 7(b) where the $\mathrm{H}-\mathrm{H}$ molecular bond is broken and each adsorbed $\mathrm{Pt}$ atom formed $\mathrm{Pt}-\mathrm{H}$ bonds with $d_{\mathrm{Pt}-\mathrm{H}}=1.56 \AA$. Upon chemisorption $d_{\text {Pt-C }}$ increased from 2.15 to $2.36 \AA$. The dissociation process is described schematically in Fig. 7(c) by plotting the variation of the total energy $E_{T}$ as a function of $z$ for two different cases. The dashed curve corresponds to the total energy of SWNT+ $\mathrm{Pt}_{2}$ and $\mathrm{H}_{2}$ calculated for different $\mathrm{H}_{2}$-tube distance $z$ by keeping the atomic configuration at $z \rightarrow \infty$ unchanged for all $z$. The continuous curve is obtained by relaxing the atomic configuration as the value of $z$ is varied. We see that for $z<4.2 \AA E_{T}$ starts to drop upon the onset of dissociation. We note a very small barrier at about $z \sim 4.5 \AA$. Upon overcoming this energy barrier, the process is exothermic with an energy gain of $\sim 1.2 \mathrm{eV}$. The continuous curve, that ends at $(x)$, corresponds to the final equilibrium configuration. 
In Figs. 7(d) and 7(f) the adsorption of a single $\mathrm{H}_{2}$ on a Pt cluster consisting of three $\mathrm{Pt}$ atoms also results in dissociation of the molecule. As the size of the cluster increases by inclusion of the third $\mathrm{Pt}$ atom, the small potential barrier at $z \sim 4.5 \AA$ is further lowered; the binding energy increased to $1.5 \mathrm{eV}$. Also one of the Pt atoms which binds with both $\mathrm{H}$ atoms is detached from the SWNT surface. This situation confirms that Pt-SWNT bonds are weakened upon the (molecular or dissociative) adsorption of $\mathrm{H}_{2}$ to $\mathrm{Pt}^{33}$ The fact that $\mathrm{Pt}-\mathrm{Pt}$ coupling is stronger than the Pt-SWNT interaction hinders the formation of uniform Pt coverage on SWNT. ${ }^{28}$ Adsorbed Pt atoms prefer to form small clusters. Present results also imply that the adsorption of $\mathrm{H}_{2}$ enhances the Pt clustering on SWNT.

The interaction between Pd atoms adsorbed on SWNT and the $\mathrm{H}_{2}$ molecule is somehow similar to the interaction with the Pt atom. However, the case with the Pd atom leads to a relatively weaker interaction and smaller binding energies. For example, the interaction between $\mathrm{H}_{2}$ and a single Pd atom adsorbed on SWNT results in a binding between chemisorption and physisorption with a binding energy of $0.6 \mathrm{eV}$. In this case, while the $\mathrm{H}-\mathrm{H}$ bond length is stretched a bit from the normal value 0.7 to $0.8 \AA$, the C-Pd bond is stretched from 2.1 to $2.2 \AA$. Small changes after the adsorption of $\mathrm{H}_{2}$ are manifestations of the relatively weak $\mathrm{H}_{2}-\mathrm{Pd}$ interaction. In contrast to the case where $\mathrm{H}_{2}$ has dissociated upon adsorption to two Pt atoms in Fig. 7(a), two adsorbed Pd atoms give rise to the chemisorption of the $\mathrm{H}_{2}$ molecule with more stretched $\mathrm{H}-\mathrm{H}$ bonds.

\section{CONCLUSIONS}

In this work we presented a detailed analysis of the interaction between hydrogen molecules and a SWNT. We found that the binding energy between a single $\mathrm{H}_{2}$ and outer surface of a bare SWNT is very weak and the physisorption bond can easily be broken. We showed that the binding of $\mathrm{H}_{2}$ to the outer surface cannot be enhanced significantly by applying a radial deformation to increase curvature effects at the site facing the $\mathrm{H}_{2}$ molecule. In contrast, the interaction between the inner surface of the $(8,0)$ tube and $\mathrm{H}_{2}$ is repulsive; this can prevent $\mathrm{H}_{2}$ molecules from entering inside this tube that has a small radius. However, the repulsive interaction may be attractive for a larger tube radius. To promote $\mathrm{H}_{2}$ uptake on the SWNT surface we considered functionalized tubes through adsorption of foreign atoms. The binding energy of $\mathrm{H}_{2}$ on the SWNT surface did not increase signifi- cantly by the coadsorption of Li. However, the binding energy increased if $\mathrm{H}_{2}$ is directly attached to an adsorbed $\mathrm{Li}$; yet the nature of the bonding remained in physisorption.

The situation with the Pt atom, which can make chemisorption bonds with the outer surface of SWNT, is found to be interesting from the point of view of $\mathrm{H}_{2}$ storage. We showed that the $\mathrm{H}_{2}$ molecule can form chemisorption bonds with free $\mathrm{Pt}$ as well as with $\mathrm{Pt}$ adsorbed on SWNT. Single $\mathrm{H}_{2}$ adsorbed on a free Pt atom dissociates and forms two strong Pt-H bonds. On the other hand, there are two stable configurations of $\mathrm{H}_{2}$ adsorbed to $\mathrm{Pt}$ atom on SWNT: While a single $\mathrm{H}_{2}$ molecule is molecularly chemisorbed to a single $\mathrm{Pt}$ atom at the $A$ site of the SWNT surface, it can dissociate if the Pt atom is adsorbed near the hollow site. The latter configuration is favorable energetically. Even the molecular adsorption of a single $\mathrm{H}_{2}$ can turn dissociative, if the second $\mathrm{H}_{2}$ is molecularly adsorbed to the same Pt atom. The dissociation of adsorbed $\mathrm{H}_{2}$ molecules leads to the weakening of Pt-C bonds. Dissociative adsorption of a single $\mathrm{H}_{2}$ to a single $\mathrm{Pt}$ atom on the graphite surface suggests that the dissociation of $\mathrm{H}_{2}$ is also favored on SWNTs having large radius. Our analysis suggests that a single Pt adsorbed on SWNT can bind up to two $\mathrm{H}_{2}$ molecules with significant binding energies in the chemisorption range. Beyond two adsorbed $\mathrm{H}_{2}$, an additional $\mathrm{H}_{2}$ molecule may form a very weak physisorption bond. A single Pd atom adsorbed on SWNT exhibits similar effects but in a relatively weaker manner as compared to that of Pt. Certainly, even if the SWNT surface were covered uniformly by Pt or Pd atoms having almost filled $d$ shells, two (or three) $\mathrm{H}_{2}$ molecules bound to each of these heavy atoms and cannot meet the target of $6 \mathrm{wp}$ set for efficient hydrogen storage medium. The $\mathrm{H}_{2}$ uptake achieved in ideal conditions can be further reduced owing to the clustering of $\mathrm{Pt}$ or Pd atoms mediated by increased coupling among themselves or by the weakening of their bonding with SWNT as a result of $\mathrm{H}_{2}$ dissociation. However, present results revealed interesting interactions between $\mathrm{H}_{2}$ and $\mathrm{Pt}$ and bonding mechanisms which have led to similar investigations of SWNTs functionalized by other light transition metal elements having less than half-filled $d$-shell (in particular, ${ }^{34} \mathrm{Sc}, \mathrm{Ti}, \mathrm{Ni}, \mathrm{V}$, etc.) for more efficient $\mathrm{H}_{2}$ storage.

\section{ACKNOWLEDGMENTS}

S.C. acknowledges partial support from TUBA, Academy of Science of Turkey. We thank Dr. T. Senger for his critical reading of the paper, and Mr. E. Durgun for his assistance in the preparation of the manuscript.
*Electronic address: ciraci@fen.bilkent.edu.tr

${ }^{1}$ A. C. Dillon, K. M. Jones, T. A. Bekkedahl, C. H. Kiang, D. S. Bethune, and M. J. Heben, Nature (London) 386, 377 (1997).

${ }^{2}$ Y. Ye, C. C. Ahn, C. Witham, B. Fultz, J. Liu, A. G. Rinzler, D. Colbert, K. A. Simith, and R. E. Smalley, Appl. Phys. Lett. 74, 16 (1999).

${ }^{3}$ C. Liu, Y. Y. Fan, M. Liu, H. T. Cong, H. M. Cheng, and M. S.
Dresselhaus, Science 286, 1127 (1999).

${ }^{4}$ S. M. Lee and Y. H. Lee, Appl. Phys. Lett. 76, 20 (2000).

${ }^{5}$ Y. Ma, Y. Xia, M. Zhao, and M. Ying, Phys. Rev. B 65, 155430 (2002).

${ }^{6}$ F. Darkrim and D. Levesque, J. Chem. Phys. 109, 12 (1998).

${ }^{7}$ K. A. Eklund and P. C. Williams, Chem. Phys. Lett. 320, 352 (2000). 
${ }^{8}$ M. Siraishi, T. Takenobu, A. Yamada, M. Ata, and H. Kataura, Chem. Phys. Lett. 358, 213 (2002).

${ }^{9}$ P. Chen, X. Wu, J. Lin, and K. L. Tan, Science 285, 91 (1999).

${ }^{10}$ P. Dubot and P. Cenedese, Phys. Rev. B 63, 241402 (2001).

${ }^{11}$ This work is based on Y. Öztürk, M.S. thesis, Bilkent University, 2003.

${ }^{12}$ P. Hohenberg and W. Kohn, Phys. Rev. 136, B864 (1964); W. Kohn and L. J. Sham, Phys. Rev. 140, A1133 (1965).

${ }^{13}$ D. Vanderbilt, Phys. Rev. B 41, R7892 (1990).

${ }^{14}$ Numerical calculations have been performed by using the VASP package: G. Kresse and J. Hafner, Phys. Rev. B 47, 558 (1993); G. Kress and J. Furthmüller, ibid. 54, 11169 (1996).

${ }^{15}$ J. P. Perdew, J. A. Chevary, S. H. Vosko, K. A. Jackson, M. R. Pederson, D. J. Singh, and C. Fiolhais, Phys. Rev. B 46, 6671 (1992).

${ }^{16}$ H. J. Monkhorst and J. D. Pack, Phys. Rev. B 13, 5188 (1976).

${ }^{17}$ W. Kohn, Y. Meir, and D. E. Makarov, Phys. Rev. Lett. 80, 4153 (1998).

${ }^{18}$ T. A. Halgren, J. Am. Chem. Soc. 114, 7827 (1992).

${ }^{19}$ E. M. Lifshitz, Zh. Eksp. Teor. Fiz. 29, 94 (1956) [Sov. Phys. JETP 2, 73 (1956)].

${ }^{20}$ In the present study we used the $(8,0)$ zigzag tube as the prototype. This tube has a small radius of $R=3.169 \AA$ and gives rise to a relatively high curvature effect. Hence, we expect that the binding energy of $\mathrm{H}_{2}$ on the $(8,0)$ tube can be an upper limit as compared to the tubes having $R>3.169 \AA$ or $n>8$.

${ }^{21}$ S. S. Han and H. M. Lee, Carbon 42, 2169 (2004). In this study $(10,0)$ SWNT is represented by a finite-length tube with end carbon atoms saturated by $\mathrm{H}$ atoms.

${ }^{22}$ As pointed out in Ref. 17, VdW interaction is poorly represented by LDA. It is even worse in the case of GGA. In the calculation of $E_{b}=E_{C}+E_{V d W}, E_{C}$ may include a small portion of $E_{V d W}$. Nevertheless, since $E_{b}$ is already small, the amount related with double counting is expected to be in the range of accuracy to be achieved by present DFT calculations.

${ }^{23}$ O. Gülseren, T. Yildirim, and S. Ciraci, Phys. Rev. Lett. 87, 116802 (2001)

${ }^{24}$ K. Tada, S. Furuya, and K. Watanabe, Phys. Rev. B 63, 155405 (2001).

${ }^{25}$ S.-P. Chan, G. Chen, X. G. Gong, and Z.-F. Liu, Phys. Rev. Lett. 87, 205502 (2001).

${ }^{26}$ O. Gülseren, T. Yildirim, S. Ciraci, and C. Kilic, Phys. Rev. B 65 , 155410 (2002); O. Gülseren, T. Yildirim, and S. Ciraci, ibid. 65, 153405 (2002)

${ }^{27}$ M. K. Kostov, M. W. Cole, and J. C. Lewis, arxiv. cond-mat/ $0007034 \mathrm{v} 1$ (unpublished). For this paper, the interaction between $\mathrm{H}_{2}$ and a SWNT surface has been calculated by using two- and three-body potentials. The range of calculated energy by Kostov et al. is in agreement with the present results.

${ }^{28}$ E. Durgun, S. Dag, V. K. Bagci, O. Gülseren, T. Yildirim, and S. Ciraci, Phys. Rev. B 67, 201401(R) (2003); E. Durgun, S. Dag, S. Ciraci, and O. Gülseren, J. Phys. Chem. B 108, 575 (2004).

${ }^{29}$ E.-C. Lee, Y.-S. Kim, Y.-G. Jin, and K. J. Chang, Phys. Rev. B 66, 073415 (2002).

${ }^{30}$ S. Dag, E. Durgun, and S. Ciraci, Phys. Rev. B 69, 121407(R) (2004); S. Dag and S. Ciraci, ibid. 71, 165414 (2005).

${ }^{31}$ X. Zhang and H. Dai, Appl. Phys. Lett. 77, 3065 (2000); Y. Zhang, N. W. Franklin, R. J. Chan, and H. Dai, Chem. Phys. Lett. 331, 35 (2000).

${ }^{32}$ Since the bond distance of $\mathrm{H}_{2}$ is extended by $20 \%$, the $\mathrm{H}-\mathrm{H}$ interaction is weaker than that in free $\mathrm{H}_{2}$. For $d_{\mathrm{H}-\mathrm{H}} \sim 1 \AA$ the adsorption may be considered as an intermediate stage between molecular and atomic adsorption.

${ }^{33}$ The calculated binding energy includes also the Pt-Pt coupling which increased after one of the Pt atoms is detached from SWNT.

${ }^{34}$ T. Yildirim and S. Ciraci, Phys. Rev. Lett. 94, 175501 (2005). 